\title{
Research on the Automatic Music Creation System based on the Human-Computer Interaction and Artificial Intelligence
}

\author{
Sui Wu \\ Hunan City University, \\ Yiyang,Hunan,413000 China
}

\begin{abstract}
In this paper, we conduct theoretical research on the automatic music creation system based on the human-computer interaction and artificial intelligence. With the rapid development of modern computer technology, the computer music software and the hardware of the agreement, the computer music has already penetrated into general music creation, production, instruments, commercial music, education, entertainment. In music education field, the use of computer music technology also has considerable development prospect. Especially music theory course in music teaching in normal colleges and universities in the use of computer music technology but a normal music professional teaching brings revolutionary role. How will the computer music technology fused into the traditional teaching mode, how to build a reasonable set of the hardware and software of the computer music teaching system is a lot of colleges and universities is a new area of exploration and practice is each care for modern music education of workers in a new topic of discussion.
\end{abstract}

Keywords- Music Creation, Human-Computer Interaction, Artificial Intelligence, Automatic.

\section{Introduction}

With the development of the society, people in material life continuously improved at the same time, the demand for basic spiritual life is becoming more and more diversity, music as people daily life indispensable additives, along with the development of the times that also faces opportunities and the challenges. In recent years, because of the change in the way music production conditions and the production, from conventional one-time recording synthesis to track; From the instinctive quality Musical Instruments playing the tape to MIDI production;
From analog to digital recording tape, etc., the express way and actual effect, not only make pop music in a short period of time to get rapid development, a large number of the musicians, producers' arises at the historic moment, the ensuing newcomer, stars make a person dazzling, people's thinking space and appreciate angle is obtained [1].

Aesthetic attention, and its physiological mechanism is to comply with the neural induction law, namely in the cerebral cortex of every excited center, can cause the inhibition of the surrounding area. In every moment, there is only one advantage excited center on cerebral cortex, the advantages of the excited area is the most easy to form a temporary connection and differentiation, and the body of all kinds of directional reflection. Music creation and appreciation of the aesthetic attention as don't like people to know, can only be a reflection of the object passive, but a complex positive and the active process. In music appreciation, due to the time limitation of art and music of aesthetic feeling, it is always building in memory, on the basis of the appearance of the generalization and representation in essence is consciousness in memory retention and retrieval. Synaesthesia can arouse the audience a wide range of thinking, with past life experiences to grasp piece of music. In general, thinking more abundant, the image of a work is to grasp the progressively more comprehensive and more profound. In this sense, the viewer is good at and gives full play to your imagination and the musicians provide music image is becoming the key of appreciator appreciation activity success or failure [2-3].

Pop music is timely, performance in the form of popular music and the mode of production is 
rich and colorful. The conclusion of the way in computer music making radical change as characteristics of the computer music production technology is simple and the powerful. With the improving of the computer performance and speed up the creation of electronic music, audio production, editing and recording and the post processing. At the same time also appeared more software synthesizer, sampler plugin and effect. The application of these products increased production effect such as the sound of the music together with its editors make convenient quick, make more and more musicians use virtual instruments of music. But the virtual instruments are often unable to show the characteristics and techniques of musical instrument itself will need to be real record. Pop music mainly includes piano, drums, guitar, etc., this article will be to the pop music production and recording techniques that were analyzed. In the figure one, we firstly show sample interface of the automatic music creation system.

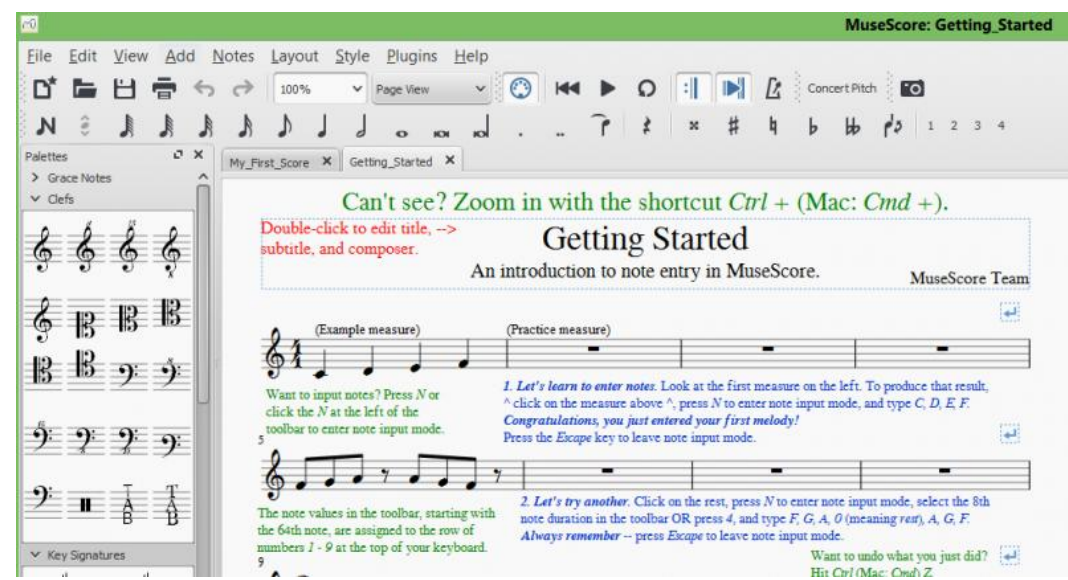

Figure 1. The Sample Interface of the Automatic Music Creation System

In this paper, we conduct theoretical research on the automatic music creation system based on the human-computer interaction and artificial intelligence. Create a high level of the premise of popular music is must have good ideas, but also must understand the characteristics of each instrument. Music production made a great contribution to music works. In today's music production technology changes with each passing day, we should continue to develop innovative music production skills, to produce more excellent works of popular music and make the greater contribution.

\section{Our Proposed Perspective}

The Principles of Human-Computer Interaction. Man-machine system is composed of interaction, mutual contact of human and machine consisting of two subsystems, to achieve specific functions and complete the task of a whole system. The edge of the man-machine system is a multidisciplinary cross science and it involves the physiology and psychology, behavioral science, cognitive science, human engineering, cybernetics and general information theory, system theory, decision science, knowledge engineering and computer science and other disciplines [4].

Ergonomics is the use of physiology, psychology and medicine and related scientific knowledge, research of the human-machine system of the machine and the human-computer interaction between people, in order to improve the efficiency of the whole system emerging edge science. Ergonomics using limit people's behavior way, the ability to work, homework etc., by means of tools, machines, systems, design task and the environment, in order to improve the safety, comfort and effectiveness of 
human-computer interaction. Early when people design of mechanical equipment, has application of the ergonomics in the design of the human-computer interaction.

The man-machine interface design principles should include the basic principles of the interface design, analysis and basic man-machine interface specification and the type of interface requirements. Interface design should first accurate classification according to user actual situation and establishes basic user type. According to the different types of users, choose the corresponding element tree and specification as think in terms of work effect, should contain the following principles. (1) Media best combination principle. Complete a task, it is necessary to use various material, following a variety of the specification requirements, should as far as possible on this basis, the best of best, choose the right material, handle the relationship between a variety of media, so as to achieve the best effect. (2) The information minimization principle. It is not the amount of information, the more the better as if you can achieve the same purpose, then, the scheme of less information is optimal in order to reduce burden of learning, memory we should use to remember the minimum information design. (3) Need to be able to cope with the user's operation command to get respond properly, so as to help users to deal with the problem. So, the system should have the ability of error recovery site design, processing to be prompt. The human-computer interaction system can be regarded as a black box, through the sensors to the environment through the effector function of the general environment. So-called environmental perception in the essence to obtain information from the basic environment around, and surrounding environment information is mutiple level, not only should know things around him, but also to know the dynamic change and development trend that is reflected from the figure one.

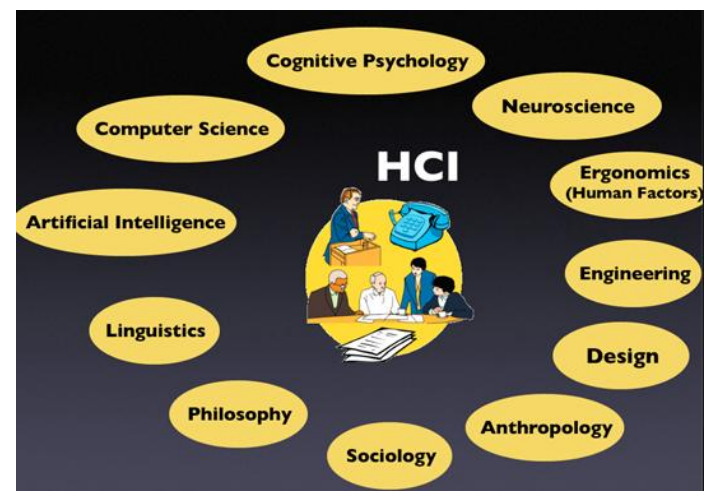

Figure 2. The Components of the HCI System with the Applications

The Characteristics of the Music Creation. In the music creation is the dynamic image thinking, its purpose primarily through the most concise statement of music vocabulary, struck a chord with the audience, so as to achieve the communication between each other. And the only way to communicate, only pay attention to the use of national music creation. This application will create a vivid image of the general music features. In national music creation, the application should hold a scientific attitude, in addition to should have the necessary writing skills as we should also pay attention to the following points. (1) Specific region amorous feelings of music works, to choose the music theme a focal point. Due to the folk music is broad, each region has the corresponding music personality, even in the same region also has a variety of music image, this request in the process of creation, fully grasp the work should reflect the style. (2) Of music works, which do not require special region in the national music selection should pay attention to the unity of the basic style and the emotional. Although there are no limitations such works, provides for the creation of a free space, but music predecessors accumulated material is music creation in the treasure, find out the commonness of melody as the motivation of the development. (3) Melt in the national music essence for yourself, who is thin hair. If, to write a good article, want to have a rich vocabulary, so to create a good melody, also 
want to have the rich music vocabulary, and rich music vocabulary comes from folk music [5-6].

The Artificial Intelligence. The research and application fields of artificial intelligence including problem solving, logical reasoning and theorem proving, natural language understanding, automatic programming, expert system, machine learning, robotics, artificial neural network (ANN), the pattern recognition, the machine vision, intelligent control, intelligent retrieval and intelligent dispatch and general command and so on. Since the emergence of artificial intelligence, the scientists in the field of research has made remarkable achievements; At the same time, the artificial intelligence research results also proved that in a particular computer can go beyond the ability of people. Of course, the core problem of artificial intelligence is still across the gap between human intelligence and machine intelligence, the problem is still has to face the artificial intelligence and the unsolved problems.

According to the literature review, the $\mathrm{Ai}$ techniques could be summarized as the listed parts. (1) Decision support system. Decision knowledge is in to the system data based on the detailed insight, analysis and speculation, so the biggest difficulty lies in the incomplete information and specification, and the nature of the data are often uncertain, and people is according to the incomplete, non-standard, uncertain information to make decisions, so the data statistical processing is an important part of any decision support system. (2) Artificial neural network. The term artificial neural network, which is relative to the biology of the biological neural network system, it is the purpose of the participant with simple mathematical model for biological neural network structure is described. This kind of general network structure and feedforward network structure there is a difference between the networks from the output layer to input layer feedback loop. (3) Expert system. Expert system is a problem solving ability with expert level in related fields of intelligent application system and it can use the domain experts for many years of accumulated experience and expertise, simulating the thinking process of the human experts, to solve the need to human experts to solve the core problem. It is the most active in artificial intelligence, one of the most promising research areas.

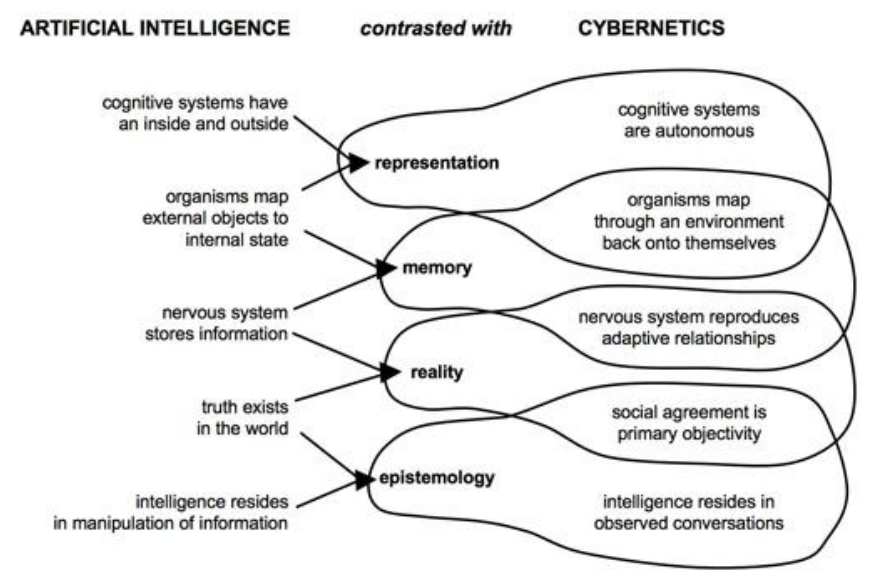

Figure 3. The Flowchart and Systematic Architecture of the AI

\section{The Automatic Music Creation System.} New technology media environment refers to transmit information through wired or the wireless network, digital information processing to use hardware equipment, special instructions to complete the program of an integrated digital work environment. The environment can be open network and terminal equipment combination of environment, can be independent digital workstation environment and also can be environment to receive the information terminal equipment and so on while no matter what kind of environment for creation of contemporary music has extensive and the revolutionary influence, many inherent in traditional patterns are great changes have taken place, and the influence and change is still in developing at a fast pace [7].

More creative group is caused by the development of the digital technology, the following software functions for their creation of learnability and general convenience. (1) Sequence audio LOOP type program. Is this software program for some Musical Instruments, 
such as guitar, bass, percussion, design the automatic playing program, set all kinds of works and style in the program. Audio LOOP directly to all kinds of musical instrument performance characteristics for short clips for the call, or by the digital synthesis of the various music audio clips. (2) Audio editing, real-time control and mixing process. This kind of program is mainly through the acquisition of music material for all kinds of the music editing and form work. (3) Online music production platform. This kind of the music program focus on online real-time interaction, is a Web based cloud music production platform, and to use the platform of virtual many audio production equipment for the use of the general creator. (4) Texture combination software programs. In this program contains the accompaniment figure, with texture prefabricated parts setting and function allocation, style, etc. Creators have to do is create a single melody and set the chords [8].

Computer music software more variety, to find, and more and more powerful and strong as the combination of the MIDI device, computer and music software, constitutes the than MIDI system function more powerful computer music system. A set of computer music system, including a more than 586 computer, through the MIDI interface connected to the keyboard, mixer, audio module, digital tape recorder, power amplifier, speakers and other external equipment, with the sequence, instruments, audio, computer simulation of mixer, effect and waveform editing tools, waveform file path, automatic program accompaniment and external digital hard disk recording all the functions, such as music and the music printing production.

\section{Conclusion}

In this paper, we conduct theoretical research on the automatic music creation system based on the human-computer interaction and artificial intelligence. Computer music because of its convenience, practical, mass, the characteristics of comprehensive, bring a lot of convenience to the music workers, its prospects will be along with the development of information technology, popularization and the promotion of faster. The author is engaged in the computer music creation and music teaching for the many years, to make music creation and music teaching of acid, sweet, bitter, hot feeling quite a lot. The rapid development of digital audio technology, the traditional music creation and production of the impact and influence, and music have different opinions or claim. In the future, we will combine more literature review and survey for advanced optimization.

\section{References}

[1] Fober, Dominique, Yann Orlarey, and Stéphane Letz. "Augmented Interactive Scores for Music Creation." Korean Electro-Acoustic Music Society's 2014 Annual Conference. 2014.

[2] Ohya, Hayato, and Satoru Morishima. "Automatic mash up music video generation system by remixing existing video content." Culture and Computing (Culture Computing), 2013 International Conference on. IEEE, 2013.

[3] Lin, Yin-Tzu, et al. "Bridging Music via Sound Effects." Multimedia (ISM), 2014 IEEE International Symposium on. IEEE, 2014.

[4] Honigman, Colin, Andrew Walton, and Ajay Kapur. "The third room: A 3d virtual music paradigm." Proceedings of the International Conference on New Interfaces for Musical Expression. 2013.

[5] Thorgersen, Ketil. "Freedom to create in the cloud or in the open? A discussion of two options for music creation with digital tools at no cost." Journal of Music, Technology \& Education 5.2 (2012): 133-144.

[6] Pilzer, Joshua D. "Music and dance in the Japanese military" comfort women" system: 
a case study in the performing arts, war, and sexual violence." Women and Music: A Journal of Gender and Culture 18.1 (2014): $1-23$.

[7] Lubin, Alex K. "Digital Music Sampling and Modern Music: The Need For The'De Minimis' Exception." (2014).
[8] Zappi, Victor, et al. "Music expression with a robot manipulator used as a bidirectional tangible interface." EURASIP J. Audio, Speech and Music Processing 2012 (2012):

2. 